\title{
Plasmid-mediated resistance to tetracyclines among Neisseria gonorrhoeae strains isolated in Poland between 2012 and 2013
}

\author{
Beata Młynarczyk-Bonikowska ${ }^{1}$, Marlena Kujawa², Magdalena Malejczyk ${ }^{1}$, Grażyna Młynarczyk ${ }^{2}$, Sławomir Majewski ${ }^{3}$ \\ 'Department of Diagnostics of Sexually Transmitted Diseases, Medical University of Warsaw, Warsaw, Poland \\ ${ }^{2}$ Department of Medical Microbiology, Medical University of Warsaw, Warsaw, Poland \\ ${ }^{3}$ Department of Dermatology and Venereology, Medical University of Warsaw, Warsaw, Poland
}

Adv Dermatol Allergol 2016; XXXIII (6): 475-479

DOI:10.5114/ada.2016.63887

\begin{abstract}
Introduction: One of two main mechanisms of resistance in tetracycline-resistant Neisseria gonorrhoeae (TRNG) is associated with the presence of TetM protein responsible for actively blocking of the tetracycline target site in the $30 \mathrm{~S}$ ribosomal subunit. This mechanism is encoded by conjugative plasmids. The second mechanism is chromosomal in nature and due to mutations in specific genes.

Aim: To determine the incidence and type of tetM determinants in TRNG strains isolated from patients presenting with gonorrhea infection to the Dermatology and Venereology Clinic in Warsaw in 2012-2013.

Material and methods: Tetracycline and doxycycline susceptibility was determined by E-Tests. The presence and type of the tet $M$ gene were determined by polymerase chain reaction.

Results: Tetracycline resistance was detected in $50.8 \%$ of the evaluated strains. The TRNG strains containing the tet $M$ plasmid constituted $13.8 \%$ of all the evaluated strains. Dutch type tet $M$ constituted $12.3 \%$ and American type tetM $1.5 \%$ of all the evaluated strains. In the remaining TRNG strains, resistance to tetracyclines was presumably chromosome-encoded. The minimal inhibitory concentration (MIC) of tetracycline ranged from 0.25 to $32.0 \mathrm{mg} / \mathrm{l}$, $\mathrm{MIC}_{50}=2.0 \mathrm{mg} / \mathrm{l}, \mathrm{MIC}_{90}=32.0 \mathrm{mg} / \mathrm{l}$. The MIC of doxycycline ranged from 0.25 to $32.0 \mathrm{mg} / \mathrm{l}, \mathrm{MIC}_{50}=4.0 \mathrm{mg} / \mathrm{l}, \mathrm{MIC}_{90}$ $=16.0 \mathrm{mg} / \mathrm{l}$.

Conclusions: Unlike most of European countries, in 2012-2013 in Poland, the Dutch type tetM was found to be much more common than the American type. Minimal inhibitory concentration values of tetracycline and doxycycline were similar, with doxycycline exhibiting a somewhat lower effectiveness in vitro than tetracycline towards chromosome-mediated tetracycline resistant strains of $N$. gonorrhoeae.
\end{abstract}

Key words: Neisseria gonorrhoeae, tetM Dutch type, tetM American type, tetracycline resistance.

\section{Introduction}

Tetracyclines are wide-spectrum antibiotics inhibiting bacterial protein synthesis via their effect on the 305 ribosomal subunit. Tetracycline resistance in different bacterial species, both Gram-negative and Gram-positive, is most commonly determined by a large group of efflux proteins, e.g. TetA to TetL belonging to the major facilitator superfamily (MFS) and by another group of proteins, such as TetM to TetW, responsible for actively blocking of the tetracycline target site in the 30 S ribosomal subunit $[1,2]$. Apart from special proteins, antibiotic resistance may be a result of a mutation in porin and transmem- brane pump regulator genes. Tetracycline resistance of Neisseria gonorrhoeae may be determined either by plasmid-encoded TetM protein or by mutations in chromosomal genes. The presence of the plasmid-encoded TetM protein typically determines the MIC of tetracycline (16-64 mg/l). Three types of conjugative plasmids have been identified in $N$. gonorrhoeae. Two of them, both of $25.2 \mathrm{MDa}$, contain the tetM gene. The third one, of 24.5 MDa, does not encode antibiotic resistance. Restriction mapping and Southern blot techniques helped to detect differences between these plasmids; consequently, the Dutch and American plasmid types were identified $[3,4]$. The tet $M$ region of both plasmid types was

Address for correspondence: Prof. Sławomir Majewski MD, PhD, Department of Dermatology and Venereology, Medical University of Warsaw, 82 A Koszykowa St, 02-008 Warsaw, Poland, phone: +48 225021313 , fax: +48 225021313 , e-mail: slawomir.majewski@wum.edu.pl Received: 22.09.2015, accepted: 28.10.2015. 
sequenced and the discovered differences served as the basis for calling the relevant tetM determinants: Dutch and American. Currently, we know that typically, though not always, the plasmid type is consistent with the tetM determinant type [5].

Tetracycline resistance in epidemic $N$. gonorrhoeae strains (e.g. G1407 genogroup) is often a result of mutations in pen B (encoding porin B) [6-9], pen C (encoding PilQ secretin) $[6,8,10]$ and overproduction of the MtrCDE efflux pump, associated with mutations in the $m \operatorname{tr} R, m \operatorname{tr} C$ promotor regions and in the $m \operatorname{tr} R$ gene [9, $11,12]$. Chromosomal mutations cause an increase in the minimal inhibitory concentration (MIC) of tetracycline, usually up to 2-4 mg/l. A comparable level of resistance is also provided by much more rare mutations in the rps) gene encoding the $\mathrm{S} 10$ ribosomal protein [13]. As a result of increasing resistance of $N$. gonorrhoeae to tetracyclines these antibiotics are not used in monotherapy to treat N. gonorrhoeae infections in Europe. However, either tetracycline or doxycycline may be used instead of azithromycin in combination with third-generation cephalosporin, especially in mixed infections, e.g. gonorrhea and chlamydia [14-17].

\section{Aim}

The purpose of our work was to determine the incidence and type of tetM determinants in TRNG strains isolated from patients presenting with gonorrhea infection to the Dermatology and Venereology Clinic in Warsaw in 2012-2013.

\section{Material and methods}

\section{Bacterial strains}

A total of $65 \mathrm{~N}$. gonorrhoeae isolates were evaluated. Nine strains were isolated from women, 56 strains were obtained from men. They had been obtained from urethral (54 strains), cervical (9 strains) or pharyngeal (2 strains) swabs of patients who visited the outpatient facility at the Dermatology and Venereology Clinic, Medical University in Warsaw at the end of 2012 and throughout 2013. Out of the strains isolated in 2012, we selected those previously unreported [18]. The strains were identified to the species level based on their colony and cell morphology as well as oxidase test and carbohydrate fermentation test results (API NH, bioMérieux). Reference N. gonorrhoeae strains ATCC ${ }^{\oplus} 49226^{\mathrm{TM}}$, ATCC $^{\oplus} 31426^{\mathrm{TM}}$ and $\mathrm{ATCC}^{\oplus} 49981^{\mathrm{TM}}$ were included. Bacteria were stored at $-70^{\circ} \mathrm{C}$ (Microbank, Fischer Scientific, USA).

\section{Susceptibility testing}

The isolated strains were tested for susceptibility to tetracycline and doxycycline using E-tests (bioMérieux, France). The tests were conducted in accordance with the manufacturer's instructions and current EUCAST guidelines. The tests were performed on plates with MuellerHinton Chocolate Agar enriched with BD IsoVitaleX ${ }^{\mathrm{TM}}$ (Becton Dickinson). E-tests were performed 10 min after inoculation and incubated in $\mathrm{CO}_{2}$-rich atmosphere (Genbox, bioMerieux) at $37^{\circ} \mathrm{C}$. Endpoint readings were conducted at $24 \mathrm{~h}$, with the use of a magnifying glass. The results were interpreted according to EUCAST and CLSI guidelines $[19,20]$.

\section{DNA isolation and PCR}

DNA was isolated with the Genomic Mini kit (A\&A Biotechnology, Poland), using the manufacturerrecommended reagents and procedure. $C 1000^{\mathrm{TM}}$ Thermal Cycler (BIO-RAD) was used for polymerase-chain reactions (PCR). Each 50- $\mu$ l sample contained $2 \mu \mathrm{l}$ of analyzed DNA, $5 \mu \mathrm{l}$ 10x buffer (MBI Fermentas), $5 \mu \mathrm{l} 1.5 \mathrm{mM} \mathrm{MgCl}$, (MBI Fermentas), $0.2 \mu$ of Taq polymerase (1.25 U, MBI Fermentas), $2 \mu \mathrm{l} 200 \mu \mathrm{M}$ MdNTPS (MBI Fermentas), $2 \mu \mathrm{l}$ $25 \mu \mathrm{M}$ solution of each of the primers (oligo.pl, IBB PAN, Warsaw), and $32.8 \mu$ l of deionized $\mathrm{H}_{2} \mathrm{O}$. The following PCR protocol was used: $94^{\circ} \mathrm{C}, 3 \mathrm{~min} ; 30$ cycles at $94^{\circ} \mathrm{C}, 20 \mathrm{~s}$ annealing, $55^{\circ} \mathrm{C}, 60 \mathrm{~s}, 72^{\circ} \mathrm{C}, 90 \mathrm{~s}, 72^{\circ} \mathrm{C}, 7 \mathrm{~min}$. We used previously reported primers [21]: UF 5'-CTCGAACAAGAGGAAGC-3'; AR 5'-GCATTCCACTTCCCAAC-3'; DR 5'-TGCAGCAGAGGGAGG -3'. Predicted PCR product sizes: UF+AR 778 bp (American type tetM), UF+DR 443 bp (Dutch type tetM). PCR products were visualized by $1 \%$ agarose gel electrophoresis (Bio-Rad) alongside GeneRuler 1kb DNA Ladder (Fermentas). Ethidium bromide-stained PCR products were visualized with Gel DOC ${ }^{\mathrm{T} M}$ XR+ Imaging System (BIO-RAD).

\section{Results}

Thirty-three (50.8\%) out of the 65 evaluated N. gonorrhoeae strains were found to be tetracycline resistant according to both EUCAST and CLSI criteria. Table 1 shows MIC value distribution and result interpretation according to EUCAST and CLSI. Table 2 presents the $M I C_{50}$ and $\mathrm{MIC}_{90}$ values of tetracycline and doxycycline. In light of no existing criteria for interpretation, susceptibility testing results for doxycycline are not presented here. Tetracycline-resistant strains were mainly (72.8\%) those with tetracycline MIC of 2-4 mg/l consistent with chromosome-mediated resistance, whereas strains with tetracycline MIC of 16-32 mg/l consistent with the plasmid tet $M$ determinant constituted $21.2 \%$ of TRNG strains. We also assessed the presence and type of the plasmid tet $M$ determinant. The results are shown in Table 3. The plasmid tetM determinant was found in 9 out of 33 TRNG strains (27.3\%), which constituted $13.8 \%$ of all evaluated $N$. gonorrhoeae strains. Out of the 9 strains with the tetM determinant, 8 (88.9\%) were of the Dutch type, and only 1 (11.1\%) exhibited the American-type determinant. None of the evaluated strains exhibited either two different 
Table 1. Tetracycline MIC values determined for 65 evaluated $N$. gonorrhoeae strains

\begin{tabular}{|c|c|c|c|c|c|}
\hline \multirow[t]{2}{*}{$\begin{array}{l}\text { Concentration } \\
{[\mathrm{mg} / \mathrm{l}]}\end{array}$} & \multirow[t]{2}{*}{ Resistance type } & \multirow{2}{*}{$\begin{array}{c}\text { Tetracycline MIC } \\
N(\%) \\
\text { of strains }\end{array}$} & \multirow{2}{*}{$\begin{array}{c}\text { Doxycycline } M \mathrm{MI}^{*} \\
N(\%) \\
\text { of strains }\end{array}$} & \multicolumn{2}{|c|}{$\begin{array}{l}\text { Tetracycline-susceptibility } \\
\text { interpretation }\end{array}$} \\
\hline & & & & EUCAST & CLSI \\
\hline 0.25 & & $1(1.5)$ & $2(3.1)$ & $\mathrm{S}$ & $\mathrm{S}$ \\
\hline 0.5 & & $5(7.7)$ & $5(7.7)$ & S & I \\
\hline 1.0 & & $26(40.0)$ & $16(24.6)$ & 1 & 1 \\
\hline 2.0 & Chromosomal & $20(30.8)$ & $10(15.4)$ & $R$ & $R$ \\
\hline 4.0 & Chromosomal & $4(6.1)$ & $23(35.4)$ & $R$ & $R$ \\
\hline 8.0 & Chromosomal & $0(0)$ & $1(1.5)$ & $R$ & $R$ \\
\hline 16.0 & tetM & $2(3.1)$ & $7(10.8)$ & $R$ & $R$ \\
\hline 32.0 & tetM & $7(10.8)$ & $1(1.5)$ & $\mathrm{R}$ & $\mathrm{R}$ \\
\hline
\end{tabular}

MIC - minimal inhibitory concentration, CLSI - Clinical and Laboratory Standards Institute, EUCAST - European Committee on Antimicrobial Susceptibility Testing, $S$ - susceptible, I - intermediate, $R$ - resistant, *no breakpoints.

Table 2. Tetracycline and doxycycline $\mathrm{MIC}_{50}$ and $M \mathrm{IC}_{90}$ for N. gonorrhoeae

\begin{tabular}{|c|c|c|c|c|c|c|c|}
\hline \multirow[t]{2}{*}{ Antibiotic } & \multirow{2}{*}{$\begin{array}{c}\text { MIC range } \\
{[\mathrm{mg} / \mathrm{ll}]}\end{array}$} & \multirow{2}{*}{$\begin{array}{l}\mathrm{MIC}_{50} \\
{[\mathrm{mg} / \mathrm{ll}]}\end{array}$} & \multirow{2}{*}{$\begin{array}{l}\mathrm{MIC}_{90} \\
{[\mathrm{mg} / \mathrm{l}]}\end{array}$} & \multirow[t]{2}{*}{ Criteria } & \multicolumn{3}{|c|}{$N(\%)$ of strains } \\
\hline & & & & & S & I & $R$ \\
\hline \multirow[t]{2}{*}{ Tetracycline } & $0.25-32.0$ & 2.0 & 32.0 & EUCAST & $6(9.2)$ & $26(40.0)$ & $33(50.8)$ \\
\hline & & & & CLSI & $1(1.5)$ & $31(47.7)$ & $33(50.8)$ \\
\hline
\end{tabular}

Doxycycline $\quad 0.25-32.0 \quad 4.0 \quad 16.0$

MIC - minimal inhibitory concentration, CLSI - Clinical and Laboratory Standards Institute, EUCAST - European Committee on Antimicrobial Susceptibility Testing, $S$ - susceptible, I-intermediate, $R$-resistant, *no breakpoints.

tetM determinants or a tetM plasmid together with a penicillinase plasmid.

\section{Discussion}

The prevalence of tetracycline resistance in N. gonorrhoeae depends on the time period and the country of strain isolation. In 2013, in India there were $12 \%$ of tetracycline resistant strains [22]; in 2010-2012, in Sri Lanka, 16.3\% [23]; in 2010-2011/2012/2013, in Belarus it was 36/35/40\%, respectively [24]; in 2010-2011, in Germany the percentage was $41.3 \%$ [25]; similarly in 2010/2011/2012, in Poland resistant strains constituted 42.9/38/49\%, respectively [18]; in 2010-2012, in Indonesia $100 \%$ strains were resistant to tetracycline [23]. Studies on the worldwide prevalence of Dutch and American type conjugative plasmids encoding the TetM protein in $N$. gonorrhoeae strains showed the Dutch type plasmids to be more prevalent in Asian countries such as Indonesia (100\%), Philippines (100\%), and Thailand (100\%) in 1988-1995 [21], in China in 1999-2006 (99.2\%) [26] and in 2011-2012 (96.2\%) [27], in Bangladesh (98.7\%) [28], in Brazil (76.5\%), in Guyana (61.1\%), in Trinidad (95.5\%), in Saint Vincent (93.3\%) [29, 30], whereas the American type plasmids were more prevalent in Europe in 1988-1995 (80.5\%) [21], in the UK in 1988-1995 (81.8\%) [21], in Italy in 2003-2005 (77.8\%) [31], in several African countries in 1988-1995 (98.3\%) [21], and in Jamaica in
Table 3. The tetM determinant type in $N$. gonorrhoeae strains

\begin{tabular}{lccc}
\hline Strain & Tetracycline MIC & Doxycycline MIC & tetM type \\
\hline 1 & 16.0 & 8.0 & Dutch \\
\hline 2 & 32.0 & 16.0 & Dutch \\
\hline 3 & 32.0 & 16.0 & Dutch \\
\hline 4 & 32.0 & 16.0 & Dutch \\
\hline 5 & 16.0 & 16.0 & American \\
\hline 6 & 32.0 & 16.0 & Dutch \\
\hline 7 & 32.0 & 32.0 & Dutch \\
\hline 8 & 32.0 & 16.0 & Dutch \\
\hline 9 & 32.0 & 16.0 & Dutch \\
\hline
\end{tabular}

1988-1995 (63\%), with a total Caribbean prevalence of $64.3 \%$ [21]. Our study showed that TetM-synthesizing N. gonorrhoeae strains isolated in Poland in 2013 are more often of the tetM Dutch type (88.9\%). Meanwhile, we observe an increase in the prevalence of the tet $M$ determinant in different countries. For example, the prevalence of tet $M$ determinant in $N$. gonorrhoeae strains in China increased 18-fold in 1999-2005 (from 1.8\% in 1999 to $32.8 \%$ in 2006) [26]. In Poland the prevalence of tetM in N. gonorrhoeae strains was $17.9 / 17.4 / 6.1 \%$ in 2010/2011/2012, respectively. 
In recent years, tetracycline-resistance of N. gonorrhoeae isolates in Poland has remained at the same high level, with approximately $1 / 3$ of isolates exhibiting plasmid-mediated resistance. Unlike in many other European countries, in Poland the prevalence of the Dutch type of tetM was much higher than that of the American type in 2012-2013. The reason of the observed difference is unknown. It does not seem to come from neighboring eastern countries either, because although the percentage of Dutch and American type of tetM in Russia was not investigated, tetM accounts only for $3 \%$ of $N$. gonorrhoeae tetracycline resistance in this country [32]. We have no data about tetM prevalence in Belarus and Ukraine, however the percentage of resistant strains in Belarus was about 40 [24]. The observed pattern of tetracycline resistance seems to be regional to Poland. The comparison with NG-MAST types of the previously isolated strains (data published before) [18] revealed that the tetM determinant was most often found in NG-MAST sequence type (ST) 1405. The type of the tetM gene was not determined at that time. The ST 1405 was the most prevalent type in Poland in 2010, 2011 and at the beginning of 2012 it was still relatively common, although it was partially replaced by epidemic ST 1407. Unlike 1407 that prevails in many European countries, ST 1405 hardly ever occurs outside Poland.

The MIC values for tetracycline and doxycycline were comparable, with doxycycline showing a slightly lower activity in vitro against TRNG not possessing tetM determinant, with tetracycline-resistance most likely to be chromosome mediated.

\section{Acknowledgments}

The work was funded by the Medical University of Warsaw. The study was conducted in the Department of Dermatology and Venereology of Medical University of Warsaw and Department of Medical Microbiology of Medical University of Warsaw.

\section{Conflict of interest}

The authors declare no conflict of interest.

\section{References}

1. Roberts MC. Environmental macrolide-lincosamide-streptogramin and tetracycline resistant bacteria. Front Microbiol 2011; 2: 40.

2. Młynarczyk B, Młynarczyk A, Kmera-Muszyńska M, et al. Mechanisms of resistance to antimicrobial drugs in pathogenic Gram-positive cocci. Mini Rev Med Chem 2010; 10 928-37.

3. Chalkley LJ, Janse van Rensburg MN, Matthee PC, et al. Plasmid analysis of Neisseria gonorrhoeae isolates and dissemination of tetM genes in southern Africa 1993-1995. J Antimicrob Chemother 1997; 40: 817-22.
4. Gascoyne DM, Heritage J, Hawkey PM, et al. Molecular evolution of tetracycline-resistance plasmids carrying TetM found in Neisseria gonorrhoeae from different countries. J Antimicrob Chemother 1991; 28: 173-83.

5. Pachulec E, Van der Does C. Conjugative plasmids of Neisseria gonorrhoeae. PLoS One 2010; 5: e9962.

6. Chen CC, Yen MY, Wong WW, et al. Tracing subsequent dissemination of a cluster of gonococcal infections caused by an ST1407-related clone harbouring mosaic penA alleles in Taiwan. J Antimicrob Chemother 2013; 68: 1567-71.

7. Jeverica S, Golparian D, Maticic M, et al. Phenotypic and molecular characterization of Neisseria gonorrhoeae isolates from Slovenia, 2006-12: rise and fall of the multidrugresistant NG-MAST genogroup 1407 clone? J Antimicrob Chemother 2014; 69: 1517-25.

8. Lee SG, Lee H, Jeong SH, et al. Various penA mutations together with mtrR, porB and ponA mutations in Neisseria gonorrhoeae isolates with reduced susceptibility to cefixime or ceftriaxone. J Antimicrob Chemother 2010; 65: 669-75.

9. Thakur SD, Starnino S, Horsman GB, et al. Unique combined penA/mtrR/porB mutations and NG-MAST strain types associated with ceftriaxone and cefixime MIC increases in a 'susceptible' Neisseria gonorrhoeae population. J Antimicrob Chemother 2014; 69: 1510-6.

10. Whiley DM, Jacobsson S, Tapsall JW, et al. Alterations of the pilQ gene in Neisseria gonorrhoeae are unlikely contributors to decreased susceptibility to ceftriaxone and cefixime in clinical gonococcal strains. J Antimicrob Chemother 2010; 65: 2543-47.

11. Hess D, Wu A, Golparian D, et al. Genome sequencing of a Neisseria gonorrhoeae isolate of a successful international clone with decreased susceptibility and resistance to extended-spectrum cephalosporins. Antimicrob Agents Chemother 2012; 56: 5633-41.

12. Ohneck EA, Zalucki YM, Johnson PJ, et al. A novel mechanism of high-level, broad-spectrum antibiotic resistance caused by a single base pair change in Neisseria gonorrhoeae. Mbio 2011; 2: e00187-11.

13. Hu M, Nandi S, Davies C, et al. High-level chromosomally mediated tetracycline resistance in Neisseria gonorrhoeae results from a point mutation in the rps) gene encoding ribosomal protein $\mathrm{S} 10$ in combination with the $\mathrm{mtrR}$ and penB resistance determinants. Antimicrob Agents Chemother 2005; 49: 4327-34.

14. Barbee LA, Soge OO, Holmes KK, et al. In vitro synergy testing of novel antimicrobial combination therapies against Neisseria gonorrhoeae. I Antimicrob Chemother 2014; 69: 1572-78.

15. Kerani RP, Stenger MR, Weinstock H, et al. Gonorrhea treatment practices in the STD Surveillance Network, 2010-2012. Sex Transm Dis 2015; 42: 6-12.

16. Lechtenberg RJ, Samuel MC, Bernstein KT, et al. Variation in adherence to the treatment guidelines for Neisseria gonorrhoeae by clinical practice setting, California, 2009 to 2011. Sex Transm Dis 2014; 41: 338-44.

17. Singh AE, Gratrix J, Martin I, et al. Gonorrhea treatment failures with oral and injectable expanded spectrum cephalosporin monotherapy vs dual therapy at 4 canadian sexually transmitted infection clinics, 2010-2013. Sex Transm Dis 2015; 42: 331-6.

18. Mtynarczyk-Bonikowska B, Serwin AB, Golparian D, et al. Gonorrhoea epidemiology, antimicrobial susceptibility/resistance and genetic characteristics of Neisseria gonorrhoeae isolates from Poland, 2010-2012. BMC Infect Dis 2014; 14: 65. 
19. European Committee on Antimicrobial Susceptibility Testing. Breakpoints tables for interpretation of MICs and zones diameters. 2015; Version 5, http://www.eucast.org.

20. Clinical and Laboratory Standards Institute (CLSI). Performance Standards for Antimicrobial Susceptibility Testing. Twenty-Fourth Informational Supplement. CLSI document M100-S24. Wayne, PA: Clinical and Laboratory Standards Institute 2014; 34: 1-220.

21. Turner A, Gough KR, Leeming JP. Molecular epidemiology of tetM genes in Neisseria gonorrhoeae. Sex Transm Infect 1999; 75: 60-6.

22. Bharara T, Bhalla P, Rawat D, et al. Rising trend of antimicrobial resistance among Neisseria gonorrhoeae isolates and the emergence of $\mathrm{N}$. gonorrhoeae isolate with decreased susceptibility to ceftriaxone. Indian J Med Microbiol 2015; 33: 39-42.

23. Bala M, Kakran M, Singh V, et al. Monitoring antimicrobial resistance in Neisseria gonorrhoeae in selected countries of the WHO South-East Asia Region between 2009 and 2012: a retrospective analysis. Sex Transm Infect 2013; 89: iv28-35.

24. Lebedzeu F, Golparian D, Titov L, et al. Antimicrobial susceptibility/resistance and NG-MAST characterisation of Neisseria gonorrhoeae in Belarus, Eastern Europe, 2010-2013. BMC Infect Dis 2015; 15: 29.

25. Horn NN, Kresken M, Körber-Irrgang B, et al. Antimicrobial susceptibility and molecular epidemiology of Neisseria gonorrhoeae in Germany. Int I Med Microbiol 2014; 304: 586-91.

26. Su X, Jiang F, Qimuge, et al. Surveillance of antimicrobial susceptibilities in Neisseria gonorrhoeae in Nanjing, China, 1999-2006. Sex Transm Dis 2007; 34: 995-9.

27. Li S, Su X, Le W, et al. Antimicrobial susceptibility of Neisseria gonorrhoeae isolates from symptomatic men attending the Nanjing sexually transmitted diseases clinic (2011-2012): genetic characteristics of isolates with reduced sensitivity to ceftriaxone. BMC Infect Dis 2014; 14: 622.

28. Alam MA, Chowdhury ZU, Chowdhury AH, et al. Epidemic plasmids in Neisseria gonorrhoeae isolated from high-risk population in Bangladesh. Mymensingh Med J 2012; 21: 220-5.

29. Dillon JA, Li H, Sealy J, et al. Antimicrobial susceptibility of Neisseria gonorrhoeae isolates from three Caribbean countries: Trinidad, Guyana, and St. Vincent. Sex Transm Dis 2001; 28: 508-14.

30. Dillon JA, Rubabaza JP, Benzaken AS, et al. Reduced susceptibility to azithromycin and high percentages of penicillin and tetracycline resistance in Neisseria gonorrhoeae isolates from Manaus, Brazil. Sex Transm Dis 2001; 28: 521-6.

31. Starnino S, Neri A, Stefanelli P, et al. Molecular analysis of tetracycline-resistant gonococci: rapid detection of resistant genotypes using a real-time PCR assay. FEMS Microbiol Lett 2008; 286: 16-23.

32. Borovskaya AD, Malakhova MV, Vereshchagin VA, et al. Analysis of the contribution of molecular mechanisms into formation of gonoccocal resistance to tetracycline. Bull Exp Biol Med 2007; 144: 432-7. 